\title{
Impacto en la Usabilidad de un Sistema Web Académico desarrollado con Tecnología PHP y Framework Symfony 2
}

Impact on the Usability of an Academic Web System developed with PHP Technology and Symfony2 Framework

Galuth Irene García Camacho. ${ }^{1}$, Linda Noralma Aguilar Moncayo. ${ }^{2}$, María Gabriela Moyano Jácome. ${ }^{3}$

Recibido: 21-04-2019 / Revisado: 25-05-2019 /Aceptado: 24-06-2019/ Publicado: 05-07-2019

\begin{abstract}
.
DOI: https://doi.org/10.33262/cienciadigital.v3i3.636

In the present investigation the analysis of the use of the academic web system developed for the "Academia Militar Tiwintsa" in Morona Santiago province was carried out, which was implemented using PHP technology with the Symfony2 framework, in order to identify the impact of the use of these technologies in the user's final interface. For which the academic system was subjected to quality tests applying ISO / IEC 9241-11, obtaining as a result in the effectiveness metric a percentage value of $93,64 \%$; in the efficiency metric a percentage value of $92,50 \%$; and in the satisfaction metric a percentage value of $94,40 \%$; results with which it was determined that the use of PHP technology with the Symfony2 framework allows the development of an intuitive and friendly system, motivating the daily use of it for the different academic and administrative processes of the institution, as it helps to improve the times of response and consequently the quality of customer service.
\end{abstract}

Keywords: Symfony2, Interface, Quality, Satisfaction.

\section{Resumen.}

En la presente investigación se realizó el análisis del uso del sistema académico web desarrollado para la "Academia Militar Tiwintsa" en la provincia Morona Santiago, el cual fue implementado utilizando la tecnología PHP con el framework Symfony2, con

1 Escuela Superior Politécnica de Chimborazo, Sede Morona Santiago. Macas, Ecuador. galuth.garcia@espoch.edu.ec

2 Escuela Superior Politécnica de Chimborazo, Sede Morona Santiago. Macas, Ecuador. laguilar@espoch.edu.ec

3 Escuela Superior Politécnica de Chimborazo, Sede Morona Santiago. Macas, Ecuador. maría.moyano@espoch.edu.ec 
la finalidad de identificar el impacto del uso de estas tecnologías en la interfaz final del usuario. Para lo cual el sistema académico fue sometido a pruebas de calidad aplicando la norma ISO/IEC 9241-11, obteniendo como resultado en la métrica de efectividad un valor porcentual de 93,64\%; en la métrica de eficiencia un valor porcentual de 92,50\%; y en la métrica de satisfacción un valor porcentual de 94,40\%; resultados con los que se determinó que el uso de la tecnología PHP con el framework Symfony2 permite el desarrollo de un sistema intuitivo y amigable, motivando el uso diario del mismo para los diferentes procesos académicos y administrativos de la institución, pues ayuda a mejorar los tiempos de respuesta y en consecuencia la calidad de atención al cliente

Palabras claves: Symfony2, Interfaz, Calidad, Satisfacción.

\section{Introducción.}

Empresas e instituciones públicas y privadas actualmente tienen la necesidad de contar con sistemas robustos que optimicen la ejecución de sus procesos, satisfaciendo las necesidades del negocio mediante la automatización de la información. Con el desarrollo de las aplicaciones web se ha tenido un crecimiento gigantesco en todos los ámbitos, esto ha permitido desarrollar sistemas escalables donde se integran componentes mediante el uso de diversas tecnologías web.

Las aplicaciones web no requieren actualizaciones en cada estación cliente, esto se debe a que la contienen un procedimiento de actualización eficaz y eficiente, evitan al usuario la molesta tarea de conseguir e instalar actualizaciones, tampoco irrumpen sus labores cotidianas para instalaciones o configuraciones de versiones más recientes, lo único necesario para que los usuarios tengan acceso a una información actualizada constantemente es dar un soporte de constante al servidor de aplicaciones. (Corsi M., 2014).

Además de ser un gran recurso educativo, sirve como plataforma para la publicación y divulgación de noticias e información, tramites online, presentación de la planta docente y un sinnúmero de actividades y servicios que brinda la institución. (Staffcreativa, 2014).

Con base a lo expuesto, se ha propuesto a la "Academia Militar Tiwintsa" el desarrollo de un sistema web utilizado el framework Symfony2 y programación PHP 5.0, para que esté al nivel de la mayoría de instituciones educativas en cuanto al manejo y gestión de la información de la institución, así como también este acorde al constante avance de la tecnología. El sistema servirá para automatizar procesos como: datos de matrículas, representantes, estudiantes, profesores, cursos, paralelos, asignaturas, control de asistencia, registro de notas y obtener reportes personalizados.

\section{Metodología}

Con la finalidad de presentar una respuesta óptima y oportuna para hacer frente a los requerimientos, se ha considerado la metodología de desarrollo ágil SCRUM, la cual presenta tres fases: 
- Fase de Planificación: análisis de requisitos, factibilidad, diseño de la arquitectura del sistema, diseño de la base de datos y el diseño de las interfaces de usuario.

- Fase de Desarrollo: involucra la gestación de los Sprint que componen el proyecto.

- Fase de Cierre: se realiza la evaluación y pruebas respectivas.

Para identificar los procesos que componen la gestión académica y definir los requerimientos de software, se realizó entrevistas a las personas directamente involucradas en la gestión de los procesos académicos de la institución. Posteriormente se estudió las funciones y características del entorno de desarrollo del framework Symfony2 para diseñar la arquitectura de los componentes del sistema Web basándose en el modelo MVC que presenta el framework; para definir y documentar cada etapa del desarrollo del sistema se utiliza la metodología de desarrollo ágil SCRUM, finalmente para conocer el impacto del uso de está tecnologías en el desarrollo del sistema académico se hace una evaluación para determinar si el diseño de la interfaz cumple con las métricas de usabilidad especificadas en la norma ISO/IEC 9241-11 tales como: efectividad, eficiencia y satisfacción permitiendo conocer de esta manera si el sistema es intuitivo y amigable para los usuarios.

Se entiende por: efectividad la precisión y nivel de cumplimiento con que los usuarios logran los objetivos específicos. eficiencia la precisión y nivel de cumplimiento con que los usuarios logran los objetivos en relación con los recursos utilizados; y satisfacción el grado de confort y aceptación del sistema utilizado por parte de los usuarios.

\section{Resultados}

Tabla 1. Parámetros de Medición

\begin{tabular}{cc}
\hline NIVEL DE ACEPTACIÓN & RANGO DE ACEPTACIÓN \\
\hline Alta & $0,71-1,00$ \\
Media & $0,36-0,70$ \\
Baja & $0,00-0,35$ \\
\hline
\end{tabular}

Fuente: Walter Sanchez,2011

Elaborado por: Las autoras

En la tabla 1 se puede apreciar los parámetros de medición a través de una escala cuantitativa, en un rango de 0 a 1 siendo 0 el valor más bajo y 1 el más alto. 
Tabla 2. Características y Sub características de Medición

\begin{tabular}{llc}
\hline CARACTERÍSTICA & \multicolumn{1}{c}{ SUB CARACTERÍSTICAS } & ACEPTACIÓN REQUERIDA \\
\hline & Adecuación & Alta \\
& Exactitud & Alta \\
Efectividad & Interoperabilidad & Alta \\
& Seguridad & Media \\
& Conformidad de la funcionabilidad & Alta \\
& Entendibilidad & Alta \\
& Aprendibilidad & Media \\
Satisfacción & Operabilidad & Baja \\
& Atractivo & Media \\
& Conformidad de la usabilidad & Alta \\
& Tiempo de respuesta & Media \\
& Utilización de recursos & Media \\
& Conformidad de eficiencia &
\end{tabular}

Fuente: Walter Sanchez,2011

Elaborado por: Las autoras

La tabla 2 muestra las características y sub características de medición junto con su nivel de aceptación en una escala cualitativa.

Tabla 3. Métrica Efectividad del Sistema

\begin{tabular}{ccccc}
\hline \multirow{2}{*}{$\begin{array}{c}\text { SUB } \\
\text { CARACTERÍSTICA }\end{array}$} & \multicolumn{2}{c}{ REQUERIDO } & \multicolumn{2}{c}{ OBTENIDO } \\
\cline { 2 - 5 } & MÉTRICA DE & NIVEL DE & MÉTRICA DE & NIVEL DE \\
& EVALUACIÓN & ACEPTACIÓN & EVALUACIÓN & ACEPTACIÓN \\
\hline Idoneidad & 1,00 & Alta & 1,00 & Alta \\
\hline
\end{tabular}


ISSN: 2602-8085

www.cienciadigital.org

Vol. 3, N³, p. 296-305, julio - septiembre, 2019

\begin{tabular}{lcccc}
\hline Exactitud & 1,00 & Alta & 0,96 & Alta \\
Interoperabilidad & 0,70 & Media & 0,50 & Media \\
Seguridad & 1,00 & Alta & 1 & Alta \\
$\begin{array}{l}\text { Conformidad de la } \\
\text { funcionabilidad }\end{array}$ & 0,70 & Media & 0,66 & Media \\
$\quad$ TOTAL & 4,40 & TOTAL & 4,12 & \\
$\quad \begin{array}{r}\text { PORCENTAJE } \\
\text { TOTAL }\end{array}$ & $100 \%$ & $\begin{array}{r}\text { PORCENTAJE } \\
\text { TOTAL }\end{array}$ & $93,64 \%$ & \\
\hline
\end{tabular}

Fuente: Trabajo de campo

Elaborado por: Las autoras

En la tabla 3 se puede evidenciar que la métrica de efectividad del sistema tiene un grado de aceptación medio - alto, llegando a un 4,12 de los 4,40 puntos del valor máximo a alcanzar, equivalente al 93,64\% de precisión y nivel de cumplimiento con que los usuarios logran los objetivos específicos.

Tabla 4. Evaluación de la Métrica Satisfacción

\begin{tabular}{|c|c|c|c|c|}
\hline \multirow{2}{*}{$\begin{array}{c}\text { SUB } \\
\text { CARACTERÍSTICA }\end{array}$} & \multicolumn{2}{|c|}{ REQUERIDO } & \multicolumn{2}{|c|}{ OBTENIDO } \\
\hline & $\begin{array}{l}\text { MÉTRICA DE } \\
\text { EVALUACIÓN }\end{array}$ & $\begin{array}{c}\text { NIVEL DE } \\
\text { ACEPTACIÓN }\end{array}$ & $\begin{array}{l}\text { MÉTRICA DE } \\
\text { EVALUACIÓN }\end{array}$ & $\begin{array}{c}\text { NIVEL DE } \\
\text { ACEPTACIÓN }\end{array}$ \\
\hline Entendibilidad & 1,00 & Alta & 0,90 & Alta \\
\hline Aprendibilidad & 1,00 & Alta & 0,80 & Alta \\
\hline Operabilidad & 0,70 & Media & 0,70 & Media \\
\hline Atractivo & 0,35 & Baja & 0,60 & Media \\
\hline $\begin{array}{l}\text { Conformidad de la } \\
\text { usabilidad }\end{array}$ & 0,70 & Media & 0,50 & Media \\
\hline TOTAL & 3,75 & TOTAL & 3,50 & \\
\hline $\begin{array}{c}\text { PORCENTAJE } \\
\text { TOTAL }\end{array}$ & $100 \%$ & $\begin{array}{c}\text { PORCENTAJE } \\
\text { TOTAL }\end{array}$ & $94,40 \%$ & \\
\hline
\end{tabular}

Fuente: Trabajo de campo

Elaborado por: Las autoras 
En la tabla 4 se puede evidenciar que la métrica de satisfacción del sistema tiene un grado de aceptación medio - alto, llegando a un 3,50 de los 3,75 puntos del valor máximo a alcanzar, equivalente al 94,40\% del grado de confort y aceptación del sistema utilizado por parte de los usuarios.

Tabla 5. Evaluación de la Métrica Eficiencia

\begin{tabular}{|c|c|c|c|c|}
\hline \multirow{2}{*}{$\begin{array}{c}\text { SUB } \\
\text { CARACTERÍSTICA }\end{array}$} & \multicolumn{2}{|c|}{ REQUERIDO } & \multicolumn{2}{|c|}{ OBTENIDO } \\
\hline & $\begin{array}{l}\text { MÉTRICA DE } \\
\text { EVALUACIÓN }\end{array}$ & $\begin{array}{c}\text { NIVEL DE } \\
\text { ACEPTACIÓN }\end{array}$ & $\begin{array}{l}\text { MÉTRICA DE } \\
\text { EVALUACIÓN }\end{array}$ & $\begin{array}{c}\text { NIVEL DE } \\
\text { ACEPTACIÓN }\end{array}$ \\
\hline Tiempo de respuesta & 1,00 & Alta & 0,90 & Alta \\
\hline $\begin{array}{l}\text { Utilización de los } \\
\text { recursos }\end{array}$ & 1,00 & Alta & 0,66 & Media \\
\hline $\begin{array}{l}\text { Conformidad de la } \\
\text { eficiencia }\end{array}$ & 0,70 & Media & 0,66 & Media \\
\hline TOTAL & 2,40 & TOTAL & 2,22 & \\
\hline $\begin{array}{r}\text { PORCENTAJE } \\
\text { TOTAL }\end{array}$ & $100 \%$ & $\begin{array}{r}\text { PORCENTAJE } \\
\text { TOTAL }\end{array}$ & $92,50 \%$ & \\
\hline
\end{tabular}

Fuente: Trabajo de campo

Elaborado por: Las autoras

En la tabla 5 se puede evidenciar que la métrica de eficiencia del sistema tiene un grado de aceptación medio - alto, llegando a un 2,22 de los 2,40 puntos del valor máximo a alcanzar, equivalente al $92,50 \%$ de precisión y nivel de cumplimiento con que los usuarios logran los objetivos en relación con los recursos utilizados.

Tabla 6. Evaluación de la Calidad de Usabilidad

\begin{tabular}{lcc}
\hline \multicolumn{1}{c}{ MÉTRICA } & $\begin{array}{c}\text { VALOR } \\
\text { OBTENIDO }\end{array}$ & PORCENTAJE \\
\hline Efectividad & 4,12 & $93,64 \%$ \\
Satisfacción & $.3,50$ & $94,40 \%$ \\
Eficiencia & 2,40 & $92,50 \%$ \\
\multicolumn{1}{c}{ TOTAL } & $\mathbf{9 , 8 4}$ & $\mathbf{9 3 , 2 7 \%}$ \\
\hline
\end{tabular}

Fuente: Trabajo de campo

Elaborado por: Las autoras 
La tabla 6 muestra un resumen de las métricas evaluadas para determinar el grado de calidad de usabilidad del sistema, se puede apreciar que se obtuvo un 93,27\% de aceptación comprobando de esta manera que el uso de las diferentes tecnologías para el desarrollo del mismo contribuyó para obtener un producto software funcional y de fácil manejo para los usuarios.

\section{Discusión}

Los resultados reflejan que el sistema académico web tiene un grado de aceptación Alto referente a la usabilidad, entendiendo como usabilidad a la eficacia, eficiencia y satisfacción con las cuales los usuarios consiguen objetivos específicos en un determinado contexto de uso.

Es importante considerar que el término usabilidad se utiliza algunas veces en un sentido limitado, para referirse específicamente a las características de un producto software que lo hacen de fácil uso; pero es significativo reconocer que los atributos requeridos para hacer a un software usable dependen también del contexto de uso. Así pues, la usabilidad no puede ser determinada únicamente por las características de un producto aislado.

Para medir la usabilidad es necesario descomponer los factores de eficacia, eficiencia y satisfacción, así como los aspectos relevantes del contexto de uso, en atributos que puedan ser medidos y verificados. La medida de la usabilidad esta expresada por el grado en que se han logrado los objetivos que se han planteado del sistema (eficacia), los recursos que se han consumido para lograr dichos objetivos (eficiencia) y el grado en que los usuarios encontraron aceptable el sistema (satisfacción). El contexto de uso integra las características relevantes de la tarea, usuario, equipamiento y medioambiente que influyen en la usabilidad, esto puede incluir los conocimientos, habilidades, experiencia, educación, entrenamiento, características físicas, capacidades motoras y sensoriales de los usuarios.

Las mediciones de eficacia, han reflejado la precisión y cumplimiento de los objetivos totales o parciales del sistema en uso, es así que con la generación de reportes personalizados la precisión se la ha podido medir por el número de errores ortográficos y desviaciones respecto al formato de referencia; mientras que el cumplimiento se lo ha medido por el número de palabras en el documento transcrito versus el número de palabras del documento original.

Las medidas de la eficiencia han expresado el nivel de eficacia conseguida con los recursos consumidos para ello; es decir, el esfuerzo y tiempo empleado en los diferentes procesos de manejo y gestión de la información académica y administrativa de la institución.

Las medidas de satisfacción han exteriorizado el confort y aceptabilidad del sistema por parte de los usuarios y de otras personas afectadas por su uso. El grado de satisfacción fue medido 
mediante una escala de actitudes a través de un cuestionario, permitiendo conocer comentarios negativos y positivos durante el uso del sistema, proporcionando indicadores útiles sobre la percepción de la usabilidad del sistema por parte de los operadores.

\section{Conclusiones.}

- La metodología ágil SCRUM, la tecnología PHP y el framework de desarrollo Symfony2 simplifica en gran medida el diseño y desarrollo del sistema web, debido a que poseen una gran cantidad de elementos facilitando así la implementación de componentes necesarios para llevar a cabo un proceso.

- La norma de calidad ISO/IEC 9241-11 sirvió para evaluar la usabilidad del Sistema Académico Web de la Academia Militar Tiwintsa evidenciando que el sistema cumple con las métricas de efectividad, eficiencia y satisfacción en un 93,27\% permitiendo establecer así que el sistema es intuitivo y amigable para los usuarios, además que mejora los tiempos de respuesta en los procesos del manejo de información y en consecuencia ayuda a brindar una mejor atención a los estudiantes y público en general.

- La elección de los criterios de medida para usabilidad depende de los requerimientos establecidos para todo el sistema y de las necesidades de la institución. Así, por ejemplo, puede ser necesario especificar criterios para conseguir el nivel mínimo aceptable de usabilidad o bien los criterios para alcanzar el nivel correspondiente a un objetivo óptimo.

\section{Referencias bibliográficas.}

- Abran, A. (2003). Software Product Evaluation-Quality Characteristics and Guidelines for their Use. Ottawa, Canadá [En línea]. Recuperado de: http://link.springer.com/article/10.1023/A:1025869312943.

- Andino, X. (2016). Desarrollo de un sistema académico orientado a la web para la unidad educativa "Juan de Velasco" utilizando Symfony y MySQL. Recuperado de: http://dspace.espoch.edu.ec/bitstream/123456789/6274/1/18T00662.pdf

- Blinder, F (2012). Cómo funciona y para qué sirve una aplicación web. Recuperado de: http://maestrofinanciero.com/como-funciona-y-para-que-sirve-una-aplicacion-web/.

- Cases E, (2014). Apache HTTP Server: ¿Qué es, ¿cómo funciona y para qué sirve? Madrid, España. Recuperado de: http://www.ibrugor.com/blog/apache-http-server-que-es-comofunciona-y-para-que-sirve/

- Castillas L y otros, (2015). Bases de datos en MySql, Catalunya, España [pdf]. Recuperado de: http://ocw.uoc.edu/computer-science-technology-and-multimedia/bases-de-datos/bases-dedatos/P06_M2109_02151.pdf

- Duarte M, (2011). Programación en PHP a través de ejemplos. Sevilla, España [pdf]. Recuperado de: http://servicio.uca.es/softwarelibre/publicaciones/apuntes_php

- Eguiluz, J. (2013). Desarrollo web ágil con Symfony2. 5. S.I. Easybook.

- Estayno, M y otros. (2009). Modelos y métricas para evaluar la calidad del software. Buenos Aires, Argentina. Recuperado de: http://sedici.unlp.edu.ar/handle/10915/19762

- $\quad$ ISO/IEC 9126. Titled Software Engineering - Product Quality 
- Montaño, D. (2011). SCRUM: Ensayo sobre Serum [blog]. Blogger. Recuperado de: http://scruming- soft.blogspot.com/2011/07/ensayo-sobre- scrumhtml

- Murray, R, Spiegel; \& Larry J, Stephens. (2002). Estadística. 3 a ed. México: McGraw-Hill, 2002, pp.218-264.

- Potencier, F. \& Weaver, R. Symfony 2- libro oficial. Disponible en: https://librosweb.es/libro/symfony_2_x/

- Sabana, M. (2006). PHP 5 con PostgreSQL 8. Lima- Perú: Megabyte. pp.17-18

- Sanz, J. () Las Normas Técnicas ISO 9241 y EN 29241 sobre pantallas de visualización. Centro Nacional de Nuevas Tecnologías. Madrid

- Sutherland, K. (2013). La Guía del SCRUM. Recuperado de: http://www.SCRUMguides.org/docs/SCRUMguide/v1/SCRUM-guide-es.pd 


\section{PARA CITAR EL ARTÍCULO INDEXADO.}

García Camacho, G., Aguilar Moncayo, L., \& Moyano Jácome, M. (2019). Impacto en la Usabilidad de un Sistema Web Académico desarrollado con Tecnología PHP y Framework Symfony2. Ciencia Digital, 3(3), 296-305. https://doi.org/10.33262/cienciadigital.v3i3.636



El artículo que se publica es de exclusiva responsabilidad de los autores y no necesariamente reflejan el pensamiento de la Revista Ciencia Digital.

El artículo queda en propiedad de la revista y, por tanto, su publicación parcial y/o total en otro medio tiene que ser autorizado por el director de la Revista Ciencia Digital.
